\title{
Resonant Intrinsic Spin Hall Effect in p-Type GaAs Quantum Well Structure
}

\author{
Xi Dai, ${ }^{1,2}$ Zhong Fang, ${ }^{2}$ Yu-Gui Yao, ${ }^{2}$ and Fu-Chun Zhang ${ }^{1}$ \\ ${ }^{1}$ Department of Physics, and Center of Theoretical and Computational Physics, The University of Hong Kong, Hong Kong, China \\ ${ }^{2}$ Beijing National Laboratory for Condensed Matter Physics, Institute of Physics, Chinese Academy of Sciences, \\ Beijing 100080, China
}

(Received 31 July 2005; published 1 March 2006)

\begin{abstract}
We study intrinsic spin Hall effect in $p$-type GaAs quantum well structure described by Luttinger Hamiltonian and a Rashba spin-orbit coupling arising from the structural inversion symmetry breaking. The Rashba term induces an energy level crossing in the lowest heavy hole subband, which gives rise to a resonant spin Hall conductance. The resonance may be used to identify the intrinsic spin Hall effect in experiments.
\end{abstract}

\section{DOI: 10.1103/PhysRevLett.96.086802}

The study of spin Hall effect (SHE), in which an electric field induces a transverse spin current, has recently evolved into a subject of intense research for its potential application to the information processing. The intrinsic SHE was proposed by Murakami et al. [1] in $p$-type semiconductor of a Luttinger Hamiltonian and by Sinova et al. [2] in twodimensional (2D) electron systems with Rashba spin-orbit coupling. Their works have generated a lot of theoretical activities [3-19]. Current theoretical understanding is that the intrinsic SHE does not survive in the diffusive transport in the thermodynamic limit for the 2D Rashba electron system [20] in the absence of strong magnetic fields [8,1114], but the effect appears to be robust in the 2D hole gases $[15,16], p$-doped bulk semiconductors, and the modified Rashba coupling case [17]. The earlier theoretical work on the extrinsic SHE is associated with the impurity scattering, such as the skew scattering and the side jump processes [21-23]. On the experimental side, there have been two groups reporting the observation of SHE. Kato et al. $[24,25]$ used Kerr rotation microscopy to detect and image electrically induced electron-spin polarization near the edge of a $n$-type semiconductor channel. The effect was suggested to be extrinsic based on the weak dependence on crystal orientation for the strained samples. Wunderlich et al. [26] observed the SHE in 2D hole system with spin-orbit coupling, and interpreted the effect to be intrinsic. In view of the unfamiliarity of the spin Hall transport, it will be desirable and important to experimentally identify if the observed SHE is intrinsic. Such an identification requires careful study of properties of the intrinsic SHE.

In this Letter we study the intrinsic SHE in $p$-type GaAs quantum well structure described by a Luttinger Hamiltonian with a Rashba spin-orbit coupling arising from the structural inversion symmetry breaking. We show that the Rashba term hybridizes the electronic subbands of the Luttinger Hamiltonian in a quantum well and induces energy level crossings in both the heavy and light hole subbands. Similar to the case in the anomalous Hall effect discussed previously by Jungwirth et al. [27] and by Onoda and Nagaosa [28], we show that the level crossing in these subbands, if occurring at the Fermi level, will give
PACS numbers: 73.63.Hs, 72.20.My, 72.25.-b, 75.47.-m

rise to a resonant intrinsic SHE characterized by a sharp peak and a sign change in the spin Hall conductance. We propose that this type of resonance should be observable in experiment to distinguish the intrinsic SHE from the extrinsic SHE by tuning the Rashba coupling strength and/or carrier density. The sign of the extrinsic SHE induced in the skew scattering, which dominates over the side jump process in the weak disorder limit of our interest here, depends on the sign of the impurity potential, and does not change with changing the carrier density or the Rashba coupling strength.

We consider an effective Hamiltonian for the hole doped quantum well with the structural inversion symmetry breaking, described by the Luttinger Hamiltonian with a confinement potential along the $z$ direction and an additional Rashba coupling term, $H=H_{L}-\lambda(\hat{z} \times \vec{p}) \cdot \vec{S}+$ $V(z)$, where $\lambda$ is the Rashba spin-orbit coupling, $\vec{p}$ is the momentum, and $\vec{S}=\left(S_{x}, S_{y}, S_{z}\right)$ are the spin-3/2 operators. $V(z)$ is a confinement potential along the $z$ direction. For simplicity, we choose $V(z)=+\infty$ for $|z|>L$ and $V(z)=0$ otherwise. Note that we have assumed that the only effect of the structural inversion symmetry breaking is to induce a Rashba coupling term in Eq. (1). The asymmetry in $V(z)$ has been neglected, since its effect will mainly change the shape of the wave functions, which is unlikely to alter the qualitative physics we discuss below. $H_{L}$ is the Luttinger effective Hamiltonian describing the hole motion in the valence band [29],

$$
H_{L}=-\frac{\hbar^{2}}{2 m}\left[\left(\gamma_{1}+\frac{5 \gamma_{2}}{2}\right) \nabla^{2}-2 \gamma_{2}(\vec{S} \cdot \vec{\nabla})^{2}\right]
$$

where $m=9.1 \times 10^{-28}$ gram is the free electron mass, and $\gamma_{1}$ and $\gamma_{2}$ are two dimensionless parameters modeling the effective mass and spin-orbit coupling around the $\Gamma$ point. Note that the translational symmetry is broken only along the $z$ direction; the momentum $\hbar \vec{k}$ in the $x-y$ plane remains to be a good quantum number. For a given $\vec{k}, H$ can be reduced to a $1 \mathrm{D}$ effective Hamiltonian 


$$
\begin{gathered}
H_{\vec{k}}=\frac{\hbar^{2}}{2 m}\left(k^{2}-\partial_{z}^{2}\right)\left(\gamma_{1}+\frac{5 \gamma_{2}}{2}\right)+V(z) \\
-\frac{\hbar^{2} \gamma_{2}}{m}\left(S_{x} k_{x}+S_{y} k_{y}-S_{z} i \partial_{z}\right)^{2}-\lambda \hbar\left(k_{y} S_{x}-k_{x} S_{y}\right) .
\end{gathered}
$$

In the special case $k=0, S_{z}$ is a good quantum number and the eigen wave functions of $H_{\vec{k}}$ are found to be $\Psi_{\alpha n}(z)=$ $\cos \left(q_{n} z\right) \chi_{a}, n=$ odd and $\Psi_{\alpha n}(z)=\sin \left(q_{n} z\right) \chi_{a}, n=$ even with $q_{n}=n \pi / 2 L$, and $n$ being positive integers. $\chi_{a}$ is the eigenstate of $S_{z}$ with $S_{z}=a=3 / 2,-3 / 2,1 / 2,-1 / 2$. The eigenstates are twofold degenerate corresponding to the eigenvalues $E_{ \pm 3 / 2, n}=\hbar^{2} q_{n}^{2} / 2 m_{h h}$ and $E_{ \pm 1 / 2, n}=\hbar^{2} q_{n}^{2} / 2 m_{l h}$, with $m_{l h}=m /\left(\gamma_{1}+2 \gamma_{2}\right)$ and $m_{h h}=m /\left(\gamma_{1}-2 \gamma_{2}\right)$ to be the effective masses for light and heavy hole subbands, respectively. The splitting of the heavy and light hole subbands at $k=0$ is due to the $\gamma_{2}$ term in $H_{L}$. Note that the Rashba coupling term vanishes at $k=0$.

For $\vec{k} \neq 0, S_{z}$ is no longer a good quantum number, and the twofold degeneracy splits and the heavy and light hole subbands will be mixed. Two limiting cases were considered previously. One is the limit $2 k L / \pi \ll 1$, while the Rashba coupling $\lambda$ is finite. This case was studied by Schliemann and Loss [4] by the perturbation theory, which gives the value of the spin Hall conductance of the order of $9 e / 8 \pi$. In this limit, the spin Hall effect is purely contributed from the Rashba term. The other limiting case is $\lambda=$ 0 , which was considered by Bernevig and Zhang [16], who calculated the spin Hall conductance by including both the lowest heavy hole and light hole subbands. The SHE in this case is purely caused by the Luttinger type spin-orbit coupling.

Below we shall study the electronic structure of Eq. (1) at a finite $\vec{k}$ and $\lambda$. We use basis wave functions of $k=0$ eigenstates, as shown above, and apply a truncated method, in which only $N$ basis states of the lowest energies are kept. We then diagonalize $H_{\vec{k}}$ within this truncated Hilbert space by numerical means. As $N$ increases, the eigenenergies of the lowest subbands converge quickly. In Fig. 1(a) we plot the lowest four subbands in the Rashba free case, namely, $\mathrm{HH} 1, \mathrm{LH} 1, \mathrm{HH} 2$, and HH3 from the bottom to top, with a double degeneracy for each subband. Here $\mathrm{HH}$ and $\mathrm{LH}$ denote heavy hole and light hole, respectively. In our calculations, we use $\gamma_{1}=7.0, \gamma_{2}=1.9$. With this choice of the parameters, the correct band structure of the subbands are reproduced [30], and the results are in good agreement with the previous calculations using the envelope function method [31-33]. The Rashba term lifts the double degeneracy of each subband at finite $k$, as shown in Figs. 1(b) and 1(c). For $k \ll \pi / 2 L$, the energy splitting is found to be proportional to $k^{3}$ for the HH1 band and to $k$ for the LH1 subband, consistent with the previous study based on the leading order perturbation around the $\Gamma$ point [4]. With the increment of $k$, the interplay between the Rashba and Luttinger type spin-orbit couplings leads to a level crossing within the subbands. For relatively small Rashba

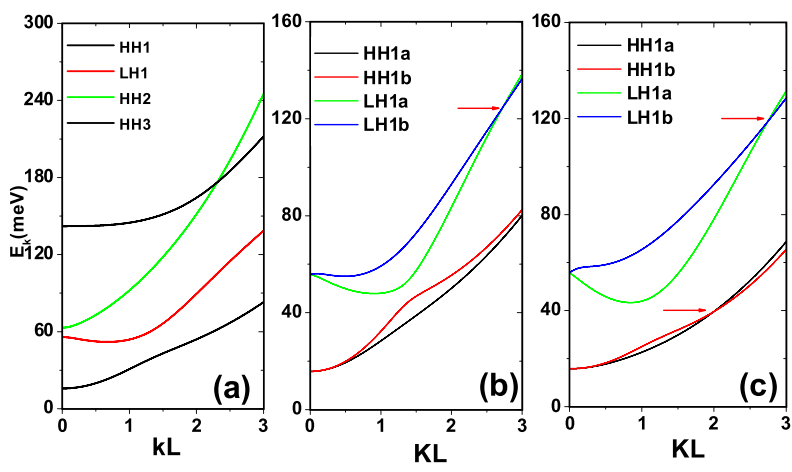

FIG. 1 (color online). Dispersion of low lying subbands for $p$-type GaAs quantum well structure with the width $2 L=83 \AA$. (a) For Rashba coupling $\lambda=0$, (b) $\lambda=\hbar^{2} / m L$, and (c) $\lambda=3.0 \times \hbar^{2} / m L$.

coupling $\left(\lambda=\hbar^{2} / m L\right)$, the level crossing occurs only in the LH1 subband. While for large Rashba coupling $(\lambda=$ $3 \hbar^{2} / m L$ ), level crossings are found in both LH1 and HH1 subbands. A careful analysis reveals that $\mathrm{HH} 2$ subband is important to the level crossings occurred in both HH1 and LH1 subbands.

We now discuss the spin Hall conductance. We consider a linear response of the spin current tensor component $j_{s, x}^{z}$ to a transverse electric field along the $y$ direction, where we define $j_{s, x}^{z}=\left(v_{x} S_{z}+S_{z} v_{x}\right) / 2$, and $v_{\alpha}=\partial H / \partial p_{\alpha}$ is the $\alpha=x$ or $y$ component of the velocity operator. The spin Hall conductance can be calculated by using Kubo formula,

$$
\sigma_{x}^{z}=-\frac{2 e}{\hbar} \int \frac{d^{2} \vec{k}}{(2 \pi)^{2}} \sum_{i^{\prime}>i} \frac{\left(f_{i^{\prime} \vec{k}}-f_{i \vec{k}}\right) \operatorname{Im}\left\langle i\left|j_{s, x}^{z}\right| i^{\prime}\right\rangle\left\langle i^{\prime}\left|v_{y}\right| i\right\rangle}{\left(E_{i^{\prime} \vec{k}}-E_{i \vec{k}}\right)^{2}}
$$

where $f$ is the Fermi distribution function, and $E_{i \vec{k}}$ is the energy of the $i$ th subband with the in-plane momentum $\vec{k}$. The calculated spin Hall conductance at zero temperature as a function of $\lambda$ for GaAs quantum well is shown in Fig. 2, where we have assumed the lifetime of carriers to be $\tau=2.0 \times 10^{-11} \mathrm{~s}$. At a lower hole density and a large $\lambda$, there is a resonance associated with the level crossing of the HH1 subbands. At a higher hole density, the resonance is associated with the level crossing of the LH1 subbands, insensitive to the value of $\lambda$. In Fig. 2 we show $\sigma_{x}^{z}$ as a function of $\lambda$ for a lower hole density case. A resonance is clearly seen at $\lambda \approx 3.15 \hbar^{2} / \mathrm{mL}$, associated with the level crossing of the HH1 subbands at the Fermi energy. The resonance becomes a singularity in $\sigma_{x}^{z}$ if we use $\tau \rightarrow \infty$ and is smoothed out if $\tau$ is 10 times smaller. The resonance may be used to identify the intrinsic SHE by tuning the Rashba coupling in experiments.

In Fig. 3 we plot the resonant Rashba coupling associated with the level crossing of the HH1 subbands as functions of hole density in GaAs quantum well for various well thickness. The parameters for the resonance appear to be accessible in experiments as we will discuss later. 


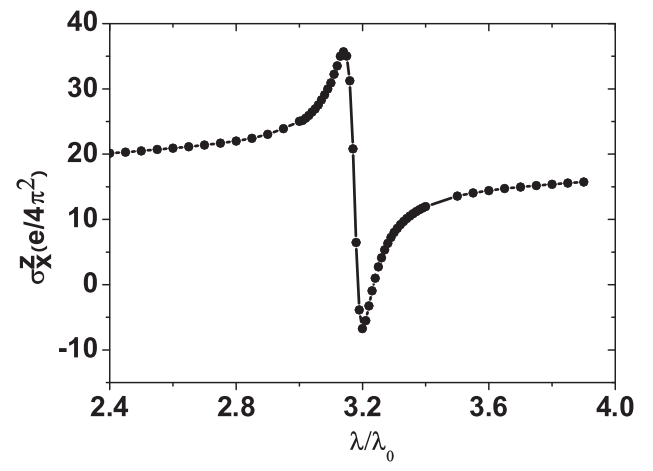

FIG. 2. Spin Hall conductance of GaAs quantum well as a function of dimensionless Rashba coupling $\lambda / \lambda_{0}$, with $\lambda_{0}=$ $\hbar^{2} / m L$. The hole density $n_{p}=5.0 \times 10^{11} / \mathrm{cm}^{2}$ and thickness $2 L=83 \AA$. A finite lifetime $\tau=2.0 \times 10^{-11} \mathrm{~s}$, equivalent to a mobility of $10^{4} \mathrm{~cm}^{2} / \mathrm{s} \mathrm{V}$, is assumed.

A few remarks are in order. The first is on the possible cancellation of the SHE due to the vertex correction. Recent theoretical works show that the vertex correction completely chancels the SHE in the 2D Rashba electron system [8] and survives in the 2D hole gas and the $p$-type bulk semiconductor [15-17]. The completely different roles the vertex corrections contribute to the SHE in the 2D electron and hole systems may be intuitively understood by symmetry consideration as argued by Bernevig and Zhang [16]. In the hole system, the effective Hamiltonian describing the HH1 subband around the $\Gamma$ point has a dispersion with a spin splitting proportional to $k^{3}$, while the dispersion of the Rashba Hamiltonian for 2D electrons has a spin splitting proportional to $k$. The current vertices for systems with $k^{3}$ and $k$ dispersions have $d$-wave and $s$-wave symmetries, respectively. Therefore, when the vertices are averaged over the $s$-wave impurity scatters, the vertex correction is zero in the hole system, but plays an important role in the electron system. The resonance we discuss here is related to the level crossing in the heavy hole subbands of the Luttinger Hamiltonian with a Rashba coupling, and the leading order of the dispersion with the spin splitting is proportional to $k^{3}$, whose current

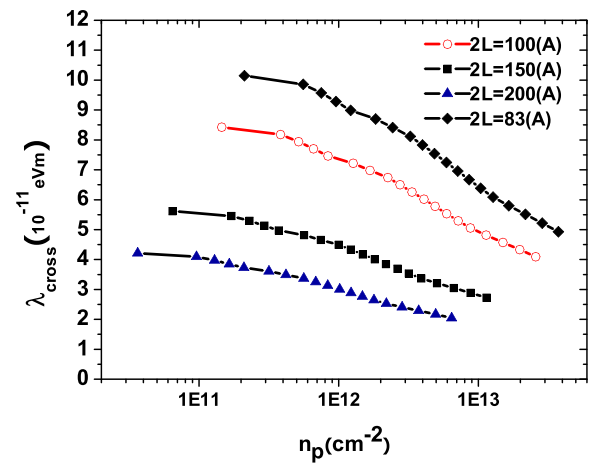

FIG. 3 (color online). The resonant Rashba coupling for the HH1 subbands as functions of hole density for various thickness $2 L$ in GaAs quantum well. vertex has $d$-wave symmetry. Therefore, we expect the resonance be robust against weak spin diffusion. Second, the resonance predicted in this system resembles the level crossings in the ferromagnetic metal with a magnetic monopolelike structure in the momentum space [34], and in the 2D quantum Hall system with a Rashba coupling [9].

In Fig. 4, we plot the spin Hall conductance as a function of the hole density for a weak Rashba coupling $\lambda=$ $\hbar^{2} / m L$. In this case, the level crossing occurs only at the LH1 subbands, and the resonant SHE appears at $n_{p}=$ $1.2 \times 10^{13} / \mathrm{cm}^{2}$. As the carrier density or chemical potential increases from zero, $\sigma_{x}^{z}$ shows two steplike features (close to arrows A and C in Fig. 4) nearby $n_{p}=0$ and at $n_{p}=4.2 \times 10^{12} / \mathrm{cm}^{2}$. They correspond to the bottoms of the HH1 and HH2 subbands, respectively, and are caused by the Rashba term. The spin Hall conductance at $n_{p} \rightarrow 0$ is found to be around $1.2 e / \pi$, which agrees well with the result obtained by Schliemann and Loss [4]. The dip feature around $n_{p}=2.5 \times 10^{12} / \mathrm{cm}^{2}$ (close to arrow B) is due to the negative effective mass of the LH1 subband near the $\Gamma$ point. The dashed line plotted in Fig. 4 is the spin Hall conductance at $\lambda=0$. The comparison shows that the resonance is contributed from the interplay between the Rashba coupling and the spin-orbit coupling in the Luttinger Hamiltonian. In terms of the Kubo formula Eq. (4), the resonance is contributed from the intrasubband transition. The robustness of the resonance associated with the LH1 subband level crossing needs more cautions. The energy splitting of the LH1 subbands is $\propto \lambda k$ at small $k$, similar to that in the Rashba electron system. Near the level crossing point, the term $\lambda k^{3}$ also becomes important. Without the $k^{3}$ term, the intrinsic SHE does not survive. It might be possible that the $k^{3}$ term discounts the vertex effect so that the SHE survives. However, this will require further study.

In the experiment of Wunderlich et al. [26], the hole density is $2.0 \times 10^{12} \mathrm{~cm}^{-2}$ and the effective width of the quantum well can be estimated to be $2 L=83 \AA$ by fitting

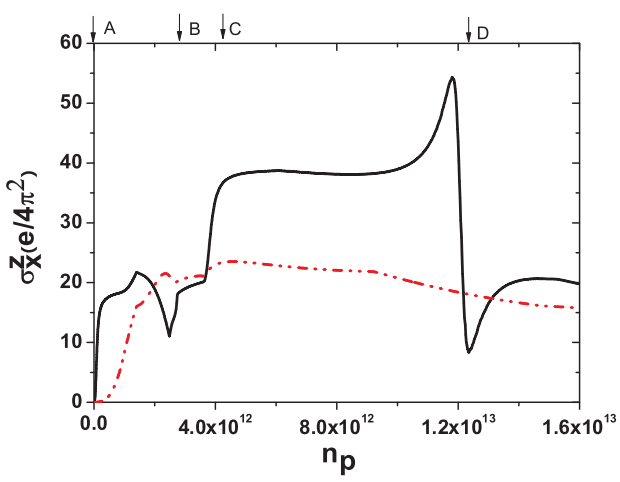

FIG. 4 (color online). Spin Hall conductance as a function of hole density for GaAs quantum well of half thickness $2 L=$ $83 \AA$ and Rashba coupling $\lambda=\hbar^{2} / m L$. Dashed line is the spin Hall conductance at $\lambda=0$. The arrows $\mathrm{A}, \mathrm{B}$, and $\mathrm{C}$ indicate the doping concentrations with which the Fermi level reaches the $\Gamma$ point value of the subbands $\mathrm{HH} 1, \mathrm{LH} 1$, and $\mathrm{HH} 2$, respectively. 
the $\Gamma$ point energy splitting of the LH1 and HH1 subbands. The Rashba coupling constant can also be extracted by fitting the splitting of the HH1 subband at the Fermi level, which is approximately $\lambda=1.5 \times 10^{-11} \mathrm{eV} \mathrm{m}$. From Fig. 3, the required Rashba coupling for the resonance is around $8.5 \times 10^{-11} \mathrm{eV} \mathrm{m}$, which is several times larger than the parameter in Wunderlich's experiment. In order to observe the resonance in the HH1 subband, one will need to either increase the Rashba coupling by about 6 times or to increase the thickness of the quantum well to approximately $200 \AA$ while keeping the 2D carrier density unchanged. Note that as shown in Fig. 2, the resonance requires high mobility (order of $10^{4} \mathrm{~cm}^{2} / \mathrm{s} \mathrm{V}$ ). The decoherence time for the 2D hole gas in the GaAs based quantum well structure can be estimated to be order of $10^{-12}$ second [32], from which we can estimate the coherence length along the $z$ direction to be around $10^{-7} \mathrm{~m}$. Because the well thickness required for the resonance is still much smaller than the typical coherence length in the system, the above conditions are likely accessible in experiments [35].

In summary, we have studied the electronic structure and the intrinsic transverse spin transport properties of the $p$-type GaAs quantum well. The Rashba spin-orbit coupling arising from the structure inversion symmetry breaking splits the subbands of the Luttinger Hamiltonian, and induces level crossings within the lowest heavy hole subbands and the lowest light hole subbands. These level crossings, if occurring at the Fermi level, give rise to resonant spin Hall conductance. Our calculations show that the parameters (the hole density, the well thickness, and the Rashba coupling strength) for the resonance are likely accessible in experiments. This phenomenon may be used to distinguish the intrinsic SHE from the extrinsic one. We expect the resonance associated with the heavy hole subbands be robust.

We have been benefited from many discussions with B. Foreman, S. Q. Shen, Z. Wang, and S. C. Zhang. This work was supported in part by Hong Kong's RGC grant and NSFC in China.

[1] S. Murakami, N. Nagaosa, and S. C. Zhang, Science 301, 1348 (2003); S. Murakami, N. Nagosa, and S.-C. Zhang, Phys. Rev. B 69, 235206 (2004).

[2] J. Sinova, D. Culcer, Q. Niu, N. A. Sinitsyn, T. Jungwirth, and A. H. MacDonald, Phys. Rev. Lett. 92, 126603 (2004).

[3] D. Culcer, J. Sinova, N. A. Sinitsyn, A. H. MacDonald, and Q. Niu, Phys. Rev. Lett. 93, 046602 (2004).

[4] J. Schliemann and D. Loss, Phys. Rev. B 69, 165315 (2004).

[5] J. Hu, B. A. Bernevig, and C. Wu, Int. J. Mod. Phys. B 17, 5991 (2003).

[6] S. Q. Shen, Phys. Rev. B 70, 081311 (2004).

[7] E. I. Rashba, Phys. Rev. B 68, 241315(R) (2003); condmat/0404723.
[8] J. Inoue, G. E. W. Bauer, and L. W. Molenkamp, Phys. Rev. B 67, 033104 (2003); 70, 041303(R) (2004).

[9] S. Q. Shen, M. Ma, X. C. Xie, and F. C. Zhang, Phys. Rev. Lett. 92, 256603 (2004).

[10] A. A. Burkov, A. S. Nunez, and A. H. MacDonald, Phys. Rev. B 70, 155308 (2004).

[11] E. G. Mishchenko, A. V. Shytov, and B. I. Halperin, Phys. Rev. Lett. 93, 226602 (2004).

[12] A. Khaetskii, Phys. Rev. Lett. 96, 056602 (2006).

[13] R. Raimondi and P. Schwab, Phys. Rev. B 71, 033311 (2005).

[14] O. Chalaev and D. Loss, Phys. Rev. B 71, 245318 (2005).

[15] J. Schliemann and D. Loss, Phys. Rev. B 71, 085308 (2005).

[16] B. A. Bernevig et al., Phys. Rev. B 70, 113301 (2004); B. A. Bervevig and S.C. Zhang, Phys. Rev. Lett. 95, 016801 (2005).

[17] S. Murakami, Phys. Rev. B 69, 241202 (2004).

[18] L. Sheng, D. N. Sheng, and C. S. Ting, Phys. Rev. Lett. 94, 016602 (2005); D. N. Sheng, L. Sheng, Z. Y. Weng, and F. D. M. Haldane, Phys. Rev. Lett. 95, 136602 (2005).

[19] K. Nomura, J. Sinova, N. A. Sinitsyn, and A.H. MacDonald, Phys. Rev. B 72, 165316 (2005).

[20] E. I. Rashba, Fiz. Tverd. Tela (Leningrad) 2, 1224 (1960) [Sov. Phys. Solid State 2, 1109 (1960)].

[21] M.I. Dyakonov and V.I. Perel, Phys. Lett. 35A, 459 (1971).

[22] J. E. Hirsch, Phys. Rev. Lett. 83, 1834 (1999).

[23] S. Zhang, Phys. Rev. Lett. 85, 393 (2000).

[24] Y. K. Kato, R.C. Myers, A.C. Gossard, and D. D. Awschalom, Science 306, 1910 (2004).

[25] V. Sih, R. C. Myers, Y. K. Kato, W. H. Kato, W. H. Lau, A.C. Gossard, and D. D. Awschalom, Nature (London), Phys. Sci. 1, 31 (2005).

[26] J. Wunderlich, B. Kaestner, J. Sinova, and T. Jungwirth, Phys. Rev. Lett. 94, 047204 (2005).

[27] T. Jungwirth, Qian Niu, and A. H. MacDonald, Phys. Rev. Lett. 88, 207208 (2002).

[28] M. Onoda and N. Nagaosa, J. Phys. Soc. Jpn. 71, 19 (2002).

[29] J. M. Luttinger, Phys. Rev. 102, 1030 (1956); R. Winkler, H. Noh, E. Tutuc, and M. Shayegan, Phys. Rev. B 65, 155303 (2002).

[30] In our calculation, a quantum well is approximated by an infinite potential well. $\gamma_{1}, \gamma_{2}$ used here are to reproduce the correct structure of the three lowest subbands in the GaAs well, which are slightly different from typical values of $\gamma_{1}=6.92, \gamma_{2}=2.1$ for the bulk GaAs. This method was adopted previously by Huang et al. [31].

[31] K. Huang, J. B. Xia, B. F. Zhu, and H. Tang, J. Lumin. 4041, 88 (1988).

[32] P. Y. Yu and M. Cardona, Fundamentals of Semiconductors (Springer, New York, 1996).

[33] M. I. Dyakonov and A. V. Khaetskii, Sov. Phys. JETP 55, 917 (1982); N. A. Merkulov, V. I. Perel, and M. E. Portnoi, Sov. Phys. JETP 72, 669 (1991).

[34] Z. Fang, N. Nagaosa, K. S. Takahashi, A. Asamitsu, R. Mathieu, T. Ogasawara, H. Yamada, M. Kawasaki, Y. Tokura, and K. Terakura, Science 302, 92 (2003).

[35] J. Wunderlich (private communication). 